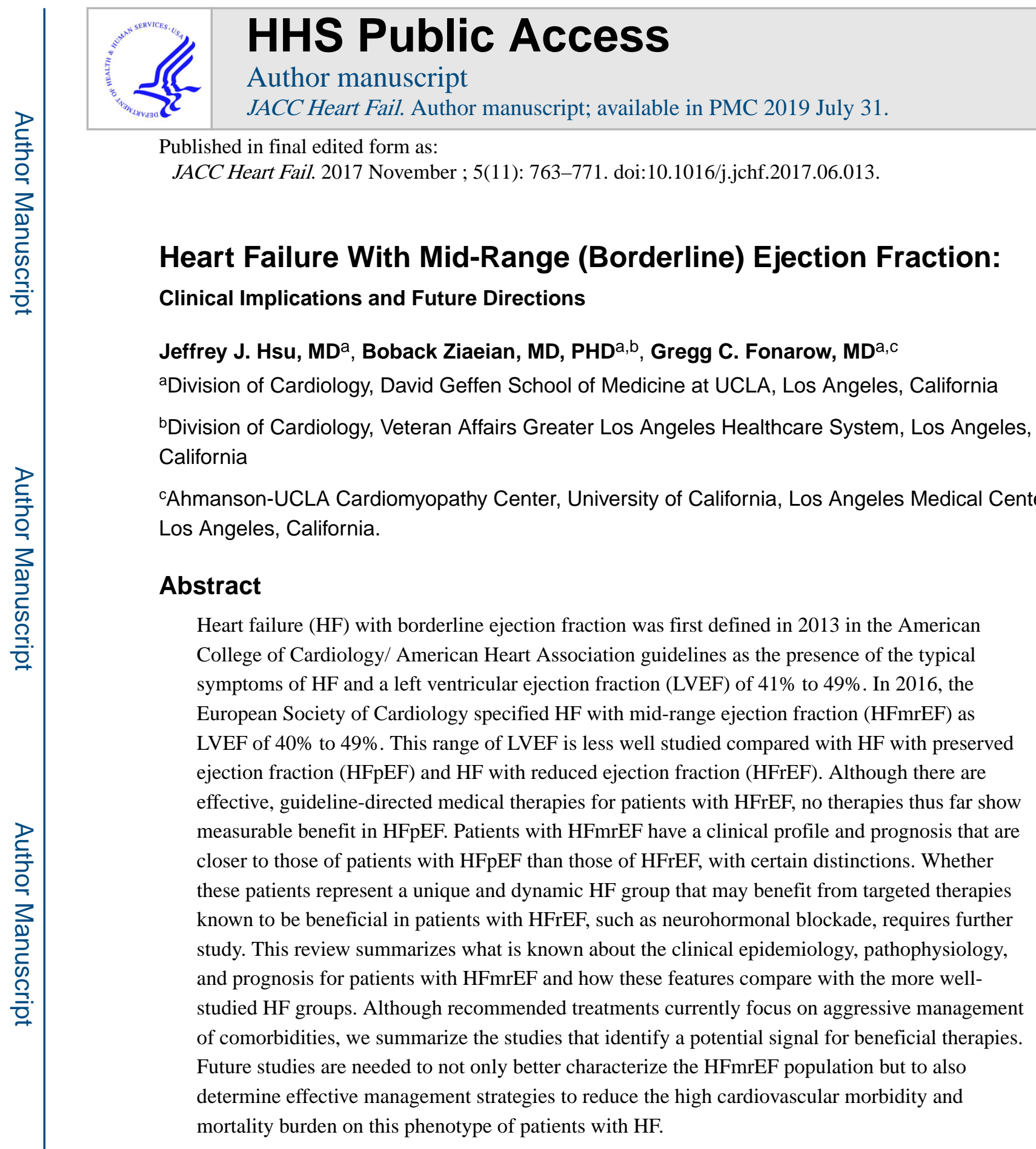

\title{
Keywords
}

epidemiology; heart failure with borderline ejection fraction; heart failure with mid-range ejection fraction; heart failure with preserved ejection fraction; HFbEF; HFmrEF; HFpEF; outcomes

The modern management of heart failure (HF) is predominantly guided by objective assessments of left ventricular ejection fraction (LVEF), which has been shown to be

ADDRESS FOR CORRESPONDENCE: Dr. Gregg C. Fonarow, Ahmanson-UCLA Cardiomyopathy Center, Ronald Reagan UCLA Medical Center, 10833 LeConte Avenue, Room A2-237 CHS, Los Angeles, California 90095-1679. gfonarow @ mednet.ucla.edu.

APPENDIX For supplemental material, please see the online version of this paper. 
predictive of adverse outcomes even in the absence of symptomatic HF (1). Historically, patients with HF have been categorized into 2 groups based on their LVEF: heart failure with reduced ejection fraction (HFrEF) and heart failure with preserved ejection fraction (HFpEF). This distinction has been important in HF management because of the different demographic characteristics, etiologies, and comorbidities between the 2 groups (2). More importantly, numerous clinical trials have shown that these groups differ in their response to therapies; although a number of medical and device therapies are proven to reduce morbidity and mortality in patients with HFrEF, there have been no therapies thus far that have been proven to improve these outcomes in patients with $\mathrm{HFpEF}$ (3).

As the field has steadily characterized important differences between these categories of HF over the years, there has been substantial variability in the LVEF ranges used to define patients with reduced and preserved EF. Studies using data from the OPTIMIZE-HF (Organized Program to Initiate Lifesaving Treatment in Hospitalized Patients with Heart Failure) and the ADHERE (Acute Decompensated Heart Failure Registry) studies began to explore the characteristics, treatment patterns, and outcomes of patients with mildly reduced LVEF, suggesting these patients may have important distinctions from those patients with HFrEF and HFpEF $(2,4)$. The most recent guidelines from the American Heart Association/ American College of Cardiology Foundation (AHA/ACCF) and the European Society of Cardiology (ESC) have defined HFrEF as having symptoms and signs of HF with an LVEF $<40 \%$, whereas HFpEF is defined as HF with an LVEF $\$ 50 \%(5,6)$. These definitions allow for a clearer distinction between the HFrEF and HFpEF groups based on LVEF, but in the process, they create an intermediate range of LVEF that has been less rigorously studied. Although the 2013 ACCF/AHA guidelines first classified this range of LVEF $41 \%$ to $49 \%$ as borderline HFpEF (5), the 2016 ESC guidelines have identified patients with HF and an LVEF $40 \%$ to $49 \%$ as having heart failure with mid-range ejection fraction (HFmrEF) (6).

Often considered a "gray" area or the "middle child" in HF (7), HFmrEF is gaining increasing attention in recent studies. Even though patients with HFmrEF have higher readmission rates than patients with HFpEF and mortality rates comparable to HFrEF and HFpEF (8), HFmrEF remains insufficiently characterized compared with the other groups. More importantly, therapies for patients with HFmrEF are unclear, as clinical trials have not directly targeted this sizeable HF population. Although much of the attention has been on HFpEF, with unsuccessful attempts at improving cardiovascular outcomes, HFmrEF may be a group primed to benefit from targeted therapies. The present review describes what is currently known about the HFmrEF population and discusses future directions to better understand and manage this vulnerable group of patients.

\section{EPIDEMIOLOGY OF HFmrEF}

\section{PREVALENCE}

More than 6.5 million people in the United States carry the diagnosis of HF and, with the aging of the population, this number will continue to rise (9). Based on recent studies, the percentage of the HF population that falls into the HFmrEF category is anywhere between $13 \%$ and $24 \%$ (10-12), suggesting that approximately 1.6 million people in the United States have HFmrEF. Analysis of temporal trends in the Get With The Guidelines-Heart 
Failure (GWTG-HF) registry have found that although the percentage of patients with HFpEF has increased (from 33\% to 39\%) and the percentage with HFrEF has decreased (from $52 \%$ to $47 \%$ ) from 2005 to 2010 , the portion with HFmrEF has remained relatively steady (between 13\% and 15\%) over this period (13).

\section{CLINICAL CHARACTERISTICS}

Determining the true clinical profile of the HFmrEF population has been challenging, as this range of LVEF is only partially included (LVEF $>45 \%$ ) or fully excluded (LVEF $>50 \%$ ) in clinical trials for HFpEF. Nonetheless, insight gained from cohort and registry studies has helped to shed light on the clinical features of this group. In 2007, an analysis of 41,267 patients in OPTIMIZE-HF analyzed patients hospitalized with HF according to LVEF group, finding that the demographic characteristics, symptom profile, comorbidities, laboratory values, and short-term outcomes of patients with LVEF between $40 \%$ and 50\% were closer to those of patients with HFpEF (2). These findings were consistent with a similar analysis performed on the ADHERE registry in 2008 comparing patients with LVEF $40 \%$ to 55\% (4). More recently, a specific analysis of the HFmrEF population was performed in $>40,000$ Medicare patients hospitalized with HF in the GWTG-HF registry, in which $14 \%$ of patients with HF fell into the HFmrEF category (8). Comparing these patients versus those in the other HF categories, patients with HFmrEF had clinical characteristics that, although intermediate between those in the HFrEF and HFpEF groups, were more similar to those of the HFpEF cohort (Table 1). These characteristics include older age, female sex, comorbidities (hypertension, chronic obstructive pulmonary disease [COPD], and diabetes mellitus [DM]), laboratory values (creatinine, B-type natriuretic peptide [BNP], and troponin), and medication use (beta-blockers, angiotensin-converting enzyme inhibitors, and angiotensin receptor blockers [ARBs]). Notably, the characteristic in which the HFmrEF population was more similar to the HFrEF population was the comorbidity of coronary artery disease (CAD) (8). These findings were consistent with the clinical profiles seen in other recent studies $(11,14)$.

\section{PATHOPHYSIOLOGY OF HFmrEF}

Studies suggest that HFrEF and HFpEF are distinct pathophysiological syndromes (15). $\mathrm{HFrEF}$ is generally characterized predominantly by systolic dysfunction and HFpEF by diastolic dysfunction, although varying degrees of overlap are often seen. Indeed, in the OPTIMIZE-HF study (2) as well as in others (16), there was a modest bimodal distribution of LVEF among patients hospitalized for HF (Figure 1), suggesting 2 distinct disease processes. However, as with HFpEF, the underlying pathophysiology of HFmrEF is not entirely clear. The ESC guidelines suggest that patients with HFmrEF likely have mild systolic dysfunction as well as diastolic dysfunction (6). A critical question is whether HFmrEF is in itself a distinct clinical syndrome or whether patients with HFmrEF are "in transition" between HFrEF and HFpEF (Online Appendix). 


\section{PROGNOSIS IN HFmrEF MORTALITY RISKS}

Mortality rates are modestly higher among patients with HFrEF but similar between those with HFmrEF and HFpEF. In OPTIMIZE-HF, the mortality rates were $3.9 \%$ for patients with HFrEF, 3.0\% for HFmrEF, and 2.9\% for HFpEF (2). Among patients in ADHERE, inhospital mortality was $4.7 \%$ in patients with LVEF <25\%, 3.4\% in patients with LVEF $25 \%$ to $40 \%, 3.2 \%$ in those with LVEF $41 \%$ to $54 \%$, and $3.0 \%$ in those with LVEF $\$ 55 \%$ (4). In a Canadian study of hospitalized patients with HF, patients with HFmrEF had unadjusted mortality rates of $5.1 \%$ at 30 days and $21.3 \%$ at 1 year (17). These 30- day and 1-year mortality rates were intermediate between those of patients with $\mathrm{HFpEF}$ (5.3\% and 22.2\%, respectively) and $\operatorname{HFrEF}$ (7.1\% and $25.5 \%$, respectively), but the differences were not statistically significant. Data from GWTG-HF found that patients with HFmrEF also had 30day and 1-year mortality rates $(8.2 \%$ and $35.1 \%)$ that were intermediate between those in the HFpEF (8.5\% and 35.6\%) and HFrEF (9.5\% and 37.5\%) groups (Figure 2) (8). In both studies, mortality rates in HFmrEF were more similar to those in the HFpEF group.

A meta-analysis of individual data from almost 40,000 patients with $\mathrm{HF}$ found that the adjusted risks of mortality steadily increased with every $5 \%$ to $10 \%$ decrease in LVEF below $40 \%$ but were not significantly different in the groups with LVEF $>40 \%$ (18). This trend was also seen in the CHARM (Candesartan in Heart Failure: Assessment of Reduction in Mortality and Morbidity) study, in which there was an adjusted hazard ratio (HR) of 1.31 for all-cause mortality per $10 \%$ reduction in LVEF $<45 \%$ (19). When comparing the predictors of mortality between HF patients with LVEF $<40 \%$ or $\$ 40 \%$, most characteristics were similarly predictive in the 2 groups (20).

More recent studies of the HFmrEF population analyzed differential characteristics that may predict increased mortality in each HF group. A recent study of the Swedish Heart Failure registry observed that chronic kidney disease was more strongly predictive of mortality in patients with HFmrEF and HFrEF than in patients with HFpEF (21). Meanwhile, in a separate study, age $\$ 85$ years and COPD were associated with a higher risk of mortality within 1 year after hospital discharge in the HFmrEF group compared with the other groups (12).

Several studies have assessed the trends of mortality rates in the different HF groups. In the GWTG- HF registry from 2005 to 2010, although unadjusted in-hospital mortality for patients with HFpEF decreased from $3.32 \%$ to $2.35 \%$, it remained relatively stable in patients with HFmrEF (2.69\% to $2.88 \%$ ) and HFrEF (3.03\% to 2.83\%) (13). In addition, an analysis of patients admitted for acute decompensated HF found that although 1-year mortality has improved slightly for all HF groups over the past approximate 20 years, longer-term mortality (2- and 5-year) has remained high (22). There were differential associations of physiological factors and comorbidities with 1-year mortality in each HF group, with age $\$ 85$ years and comorbid COPD found to be more strongly associated with 1year mortality in patients with HFmrEF (12). 


\section{MORBIDITY IN HFmrEF}

Similar to the mortality rate trends, readmission rates in HFmrEF are intermediate to those seen in HFrEF and HFpEF. In the GWTG-HF registry, all-cause readmission rates for patients with HFmrEF were $20.9 \%$ at 30 days and $63.2 \%$ at 1 year, which were similar to those seen in patients with HFpEF (20.5\% and 62.5\%) and slightly higher than those seen in patients with HFrEF (19.7\% and 59.6\%) (Figure 2) (8). However, rates of cardiovascular readmission for patients with $\operatorname{HFmrEF}$ ( $11.3 \%$ at 30 days, $41.6 \%$ at 1 year) were higher than those seen in HFpEF (9.9\% and 37.4\%) and closer to those observed in patients with HFrEF (12.9\% and 42.4\%). HF-specific readmission rates were intermediate in HFmrEF compared to those seen in HFrEF and HFpEF (8). An analysis of factors leading to HF-specific readmissions in each HF group showed that the pre-cipitants in HFmrEF (e.g., respiratory issues, uncontrolled hypertension) more closely resembled those in HFpEF, with the exception of coronary ischemia, which resembled the contributions seen in HFrEF (10).

\section{MANAGEMENT OF HFmrEF}

Contrary to HFrEF, there have been no therapies thus far that have conclusively been shown to improve outcomes in patients with HFmrEF and HFpEF (3). Thus, the recommendations from the AHA/ACCF and the ESC guidelines for the HFmrEF population currently focus on managing comorbidities and risk factors $(5,6)$. In addition, diuretic therapy is recommended to help alleviate symptoms in patients who exhibit signs of congestion. Otherwise, there are currently no specific guideline-directed medical therapies that are Class I or Class IIa recommended to improve outcomes for patients with HFmrEF.

\section{PHARMACOLOGICAL THERAPY}

The central feature of pharmacological guideline-directed medical therapy in patients with HFrEF is neurohormonal blockade with beta-adrenergic receptor blockers and inhibitors of the renin-angiotensin-aldosterone system $(5,6)$. Although these agents have not been found to be effective at improving outcomes in patients with HFpEF, they have not been specifically studied in the HFmrEF population. Nonetheless, several studies have partially or wholly included the LVEF $40 \%$ to $50 \%$ range and may help provide insight into the potential benefit of these therapies in this group.

The most recent trial of note that evaluated the effects of neurohormonal blockade in patients with HF and LVEF \$45\% was the TOPCAT (Treatment of Preserved Cardiac Function Heart Failure with an Aldosterone Antagonist) study (23). In this study of $>3,000$ patients, there was no effect of spironolactone on the composite primary outcome of cardiovascular death, aborted cardiac arrest, or HF hospitalization; however, there was a reduction in HF hospitalizations in the treatment group (HR: 0.83). Moreover, there was a significant interaction between treatment and LVEF, with stronger benefit seen in patients with LVEF $45 \%$ to $50 \%$ (24). In light of these findings from TOPCAT, the AHA/ACCF recently released an update to its guidelines, adding the Class IIb recommendation that in a selected population of patients with HF with LVEF $\geq 45 \%$, aldosterone antagonists may be considered to decrease hospitalizations (25). 
Similarly, the CHARM-Preserved trial evaluated the effects of the ARB candesartan in patients with HF with LVEF >40\% (26) and found a reduced risk of HF hospitalization in the treatment group (HR: 0.84). However, in the I-PRESERVE (Irbesartan in Heart Failure with Preserved Ejection Fraction) trial, in which the effects of irbesartan were assessed in patients with HF and LVEF $\geq 45 \%$, there were no significant benefits seen (27), although the average LVEF was notably higher in I-PRESERVE (mean LVEF: 59\%) compared with the CHARM-Preserved trial (mean LVEF: 54\%). In addition, one of the major advances to therapy for patients with HFrEF has been the coadministration of the neprilysin inhibitor sacubitril with the ARB valsartan. The ongoing PARAGON-HF (Efficacy and Safety of LCZ696 Compared to Valsartan on Morbidity and Mortality in Heart Failure Patients with Preserved Ejection Fraction) trial (NCT01920711) will compare treatment with sacubitril/ valsartan versus valsartan alone in patients with HF and LVEF $\geq 45 \%$, which may help provide some insight into the role of neprilysin inhibition in HFmrEF management.

Given the paucity of randomized clinical trial data, observational studies on the use of angiotensin-converting enzyme inhibitors/ARBs and betablockers have been undertaken. In OPTIMIZE-HF, although use of angiotensin-converting enzyme inhibitor/ARB therapy was associated with lower death and rehospitalization at 60 to 90 days in patients with HFrEF, patients with either HFmrEF or HFpEF had no associated benefit (2). Similar findings were observed for beta-blockers. The effects of beta-blockade were examined in the OPTIMIZEHF registry linked to Medicare data, and those with LVEF < $40 \%$ were compared with those with LVEF $\geq 40 \%$ for long-term outcomes (2). Although initiation of beta-blocker therapy was associated with improved outcomes in patients with HFrEF, it was not found to significantly influence outcomes in patients with LVEF $\geq 40 \%$ (28). The same findings were observed when the analysis was confined to patients with LVEF $40 \%$ to $50 \%$.

In the TIME-CHF (Trial of Intensified Versus Standard Medical Therapy in Elderly Patients With Congestive Heart Failure), N-terminal proBNP-guided management was found to be improve HF hospitalization-free survival in patients with HFmrEF and HFrEF but not in patients with HFpEF (14). However, the GUIDE-IT (Guiding Evidence Based Therapy Using Biomarker Intensified Treatment) trial also used N-terminal proBNP-guided therapy in patients with HFrEF, but this study was terminated prematurely due to futility (29). The role of biomarkers in the management of HFmrEF thus remains to be determined.

\section{MANAGEMENT OF COMORBIDITIES}

As mentioned previously, the comorbidities seen in patients with HFmrEF track more closely with those of patients with HFpEF (8). CAD has been associated with greater declines in LVEF among patients with HFpEF (30). Accordingly, screening for and management of $\mathrm{CAD}$ is a reasonable approach that may help prevent further progression of left ventricular systolic dysfunction in patients with HFmrEF, as they have been shown to have a higher rate of transitioning to HFrEF compared with patients with HFpEF (11).

Nonetheless, noncardiac comorbidities (i.e., COPD, hypertension, chronic kidney disease, DM) are highly prevalent in the HF population and contribute significantly to the overall morbidity of these patients (31). In patients with HFmrEF, uncontrolled hypertension was more often the precipitating factor for HF hospitalization compared with the other HF 
groups (10). In light of the modest signals for reduced hospitalizations with ARBs or aldosterone antagonist therapy in patients with HFpEF, these agents may be reasonable to use in efforts to manage hypertension and reduce the risk of progressive decline in LVEF in the HFmrEF population. In addition, with regard to the management of DM in patients with HF, the use of sodium glucose cotransporter 2 inhibitors in patients at high cardiovascular risk has been shown to reduce cardiovascular morbidity and mortality, including a reduction in HF hospitalization (32), and these agents are gaining favor in the management of DM in patients with HF (33).

\section{FUTURE DIRECTIONS}

Following the definition of HFmrEF by the AHA/ACCF and ESC, a number of studies have helped to elucidate some of the mysteries of this "gray" area of HF, including its prevalence, clinical characteristics, and outcomes (Central Illustration). However, as with HFpEF, there currently are no effective, guideline-directed medical therapies that improve major outcomes in this vulnerable population of patients. With the ongoing struggle of finding effective therapies for patients with HFpEF, a specific focus on and/or inclusion of patients with HFmrEF may lead to more promising results.

As discussed by Lam and Solomon (7), however, specifically targeting the HFmrEF population in clinical trials is challenging, and recent trials studying patients with the middle range of LVEF had to be stopped early due to difficulties with enrollment. In addition, the variability of LVEF measurements based on echocardiography is rather high, and the potential misclassifications of patients with HF into the different HF groups may cloud the true efficacy of therapies being studied. Thus, there are limitations to a classification system based on LVEF alone, and further refinement of the particular HF etiologies (i.e., ischemic, familial, hypertensive) and detailed pheno-typing may help to maximize the discovery of more effective treatment strategies. Whether this EF classification approach adopted in recent versions of the guidelines increases clarity, facilitates improved care, and helps move the field forward remains to be seen.

In the era of precision medicine, the next advances in the management of HFmrEF may involve identifying features of each patient with HF that can help provide further risk stratification beyond what is predicted by LVEF alone. In addition, advanced imaging modalities may identify high-risk patients within the HFmrEF group. Detection of late gadolinium enhancement on cardiac magnetic resonance imaging was found to be predictive of death or appropriate implantable cardioverter-defibrillator discharge in HF patients with LVEF $>30 \%$ (34). A recent study has shown that in patients with dilated cardiomyopathy and LVEF $\geq 40 \%$, the presence of mid-wall late gadolinium enhancement on cardiac magnetic resonance imaging was strongly predictive of the composite endpoint of sudden cardiac death or aborted sudden cardiac death (HR: 35.9) and in patients with LVEF 40\% to $50 \%$ had marked predictive value beyond that of LVEF alone (35). Thus, studies suggest the potential value of incorporating cardiac magnetic resonance imaging assessment in patients with HFmrEF and HFpEF, and further research is needed to determine whether these subgroups of patients with late gadolinium enhancement would benefit from implantable cardioverter-defibrillator placement. 
Furthermore, expansion of the tools clinicians use to refine their assessment of a patient's hemodynamic variables may improve outcomes in patients with HFmrEF. The use of an implantable microelectromechanical systems pressure sensor in the pulmonary artery to guide management of patients with HF was found to reduce hospitalizations for HF, including in those patients with LVEF $\$ 40 \%$ (36). In addition, the role of biomarkers in guiding the management of patients with HF remains an active area of investigation (37).

\section{CONCLUSIONS}

With a clearer definition of HFmrEF established by the professional guidelines, we now have a better understanding of the heterogeneous clinical profile and similarly poor outcomes of these patients compared with the other HF groups. Beyond close clinical monitoring and aggressive management of comorbidities, particularly $\mathrm{CAD}$, there may be benefit to earlier initiation of neurohormonal blockade if otherwise indicated. Further studies are needed to determine whether therapies that are ineffective in HFpEF are in fact effective in reducing morbidity and mortality in the HFmrEF population.

\section{Supplementary Material}

Refer to Web version on PubMed Central for supplementary material.

\section{Acknowledgments}

Author Disclosures: Dr. Fonarow has received research funds from the National Institutes of Health; and consulting fees from Amgen, Janssen, Medtronic, Novartis, and St. Jude Medical. All other authors have reported that they have no relationships relevant to the contents of this paper to disclose.

\section{ABBREVIATIONS AND ACRONYMS}

$\mathrm{ACCF}$

AHA

ARB

BNP

CAD

COPD

DM

ESC

GWTG-HF

HF

HFmrEF

HFpEF
American College of Cardiology Foundation

American Heart Association

angiotensin receptor blocker

B-type natriuretic peptide

coronary artery disease

chronic obstructive pulmonary disease

diabetes mellitus

European Society of Cardiology

Get With The Guidelines-Heart Failure

heart failure

heart failure with mid-range ejection fraction

heart failure with preserved ejection fraction 

HFrEF
heart failure with reduced ejection fraction
HR
hazard ratio

LVEF

left ventricular ejection fraction

\section{REFERENCES}

1. Wang TJ, Evans JC, Benjamin EJ, Levy D, LeRoy EC, Vasan RS. Natural history of asymptomatic left ventricular systolic dysfunction in the community. Circulation 2003;108:977-82. [PubMed: 12912813]

2. Fonarow GC, Stough WG, Abraham WT, et al. Characteristics, treatments, and outcomes of patients with preserved systolic function hospitalized for heart failure: a report from the OPTIMIZE-HF Registry. J Am Coll Cardiol 2007;50: 768-77. [PubMed: 17707182]

3. Butler J, Fonarow GC, Zile MR, et al. Developing therapies for heart failure with preserved ejection fraction: current state and future directions. J Am CoLL Cardiol HF 2014;2:97-112.

4. Sweitzer NK, Lopatin M, Yancy CW, Mills RM, Stevenson LW. Comparison of clinical features and outcomes of patients hospitalized with heart failure and normal ejection fraction ( $>$ or $=55 \%$ ) versus those with mildly reduced $(40 \%$ to $55 \%)$ and moderately to severely reduced $(<40 \%)$ fractions. Am J Cardiol 2008;101:1151-6. [PubMed: 18394450]

5. Yancy CW, Jessup M, Bozkurt B, et al. 2013 ACCF/AHA guideline for the management of heart failure: a report of the American College of Cardiology Foundation/American Heart Association Task Force on Practice Guidelines. J Am Coll Cardiol 2013;62:e147-239. [PubMed: 23747642]

6. Ponikowski P, Voors AA, Anker SD, et al. 2016 ESC guidelines for the diagnosis and treatment of acute and chronic heart failure: the Task Force for the diagnosis and treatment of acute and chronic heart failure of the European Society of Cardiology (ESC) developed with the special contribution of the Heart Failure Association (HFA) of the ESC. Eur Heart J 2016;37:2129-200. [PubMed: 27206819]

7. Lam CS, Solomon SD. Fussing over the middle child: heart failure with mid-range ejection fraction. Circulation 2017;135:1279-80. [PubMed: 28373521]

8. Cheng RK, Cox M, Neely ML, et al. Outcomes in patients with heart failure with preserved, borderline, and reduced ejection fraction in the Medicare population. Am Heart J 2014;168:721-30. [PubMed: 25440801]

9. Benjamin EJ, Blaha MJ, Chiuve SE, et al. Heart disease and stroke statistics-2017 update: a report from the American Heart Association. Circulation 2017;135:e146-603. [PubMed: 28122885]

10. Kapoor JR, Kapoor R, Ju C, et al. Precipitating clinical factors, heart failure characterization, and outcomes in patients hospitalized with heart failure with reduced, borderline, and preserved ejection fraction. J Am Coll Cardiol HF 2016;4:464-72.

11. Tsuji K, Sakata Y, Nochioka K, et al. Characterization of heart failure patients with mid-range left ventricular ejection fraction-a report from the CHART-2 Study. Eur J Heart Fail 2017331 [E-pub ahead of print].

12. Coles AH, Tisminetzky M, Yarzebski J, et al. Magnitude of and prognostic factors associated with 1-year mortality after hospital discharge for acute decompensated heart failure based on ejection fraction findings. J Am Heart Assoc 2015;4: e002303. [PubMed: 26702084]

13. Steinberg BA, Zhao X, Heidenreich PA, et al. Trends in patients hospitalized with heart failure and preserved left ventricular ejection fraction: prevalence, therapies, and outcomes. Circulation 2012;126:65-75. [PubMed: 22615345]

14. Rickenbacher P, Kaufmann BA, Maeder MT, et al. Heart failure with mid-range ejection fraction: a distinct clinical entity? Insights from the Trial of Intensified Versus Standard Medical Therapy in Elderly Patients With Congestive Heart Failure (TIME-CHF). Eur J Heart Fail 2017315 [E-pub ahead of print].

15. Lee DS, Gona P, Vasan RS, et al. Relation of disease pathogenesis and risk factors to heart failure with preserved or reduced ejection fraction: insights from the Framingham Heart Study of the National Heart, Lung, and Blood Institute. Circulation 2009;119:3070-7. [PubMed: 19506115] 
16. Borlaug BA, Redfield MM. Diastolic and systolic heart failure are distinct phenotypes within the heart failure spectrum. Circulation 2011;123: 2006-13; discussion 2014. [PubMed: 21555723]

17. Bhatia RS, Tu JV, Lee DS, et al. Outcome of heart failure with preserved ejection fraction in a population-based study. N Engl J Med 2006;355: 260-9. [PubMed: 16855266]

18. Meta-analysis Global Group in Chronic Heart Failure (MAGGIC). The survival of patients with heart failure with preserved or reduced left ventricular ejection fraction: an individual patient data meta-analysis. Eur Heart J 2012;33:1750-7. [PubMed: 21821849]

19. Solomon SD, Anavekar N, Skali H, et al. Influence of ejection fraction on cardiovascular outcomes in a broad spectrum of heart failure patients. Circulation 2005;112:3738-44. [PubMed: 16330684]

20. Pocock SJ, Ariti CA, McMurray JJ, et al. Predicting survival in heart failure: a risk score based on 39372 patients from 30 studies. Eur Heart J 2013;34:1404-13. [PubMed: 23095984]

21. Löfman I, Szummer K, Dahlström U, Jernberg T, Lund LH. Associations with and prognostic impact of chronic kidney disease in heart failure with preserved, mid-range, and reduced ejection fraction. Eur J Heart Fail 2017329 [E-pub ahead of print].

22. Coles AH, Fisher K, Darling C, et al. Long-term survival for patients with acute decompensated heart failure according to ejection fraction findings. Am J Cardiol 2014;114:862-8. [PubMed: 25092194]

23. Pitt B, Pfeffer MA, Assmann SF, et al. Spironolactone for heart failure with preserved ejection fraction. N Engl J Med 2014;370:1383-92. [PubMed: 24716680]

24. Solomon SD, Claggett B, Lewis EF, et al. Influence of ejection fraction on outcomes and efficacy of spironolactone in patients with heart failure with preserved ejection fraction. Eur Heart $\mathbf{J}$ 2016;37:455-62. [PubMed: 26374849]

25. Yancy CW, Jessup M, Bozkurt B, et al. 2017 ACC/ AHA/HFSA focused update of the 2013 ACCF/AHA guideline for the management of heart failure: a report of the American College of Cardiology/ American Heart Association Task Force on Clinical Practice Guidelines and the Heart Failure Society of America. J Am Coll Cardiol 2017421 [E-pub ahead of print].

26. Yusuf S, Pfeffer MA, Swedberg K, et al. Effects of candesartan in patients with chronic heart failure and preserved left-ventricular ejection fraction: the CHARM-Preserved Trial. Lancet Lond Engl 2003;362:777-81.

27. Massie BM, Carson PE, McMurray JJ, et al. Irbesartan in patients with heart failure and preserved ejection fraction. N Engl J Med 2008;359: 2456-67. [PubMed: 19001508]

28. Hernandez AF, Hammill BG, O'Connor CM, Schulman KA, Curtis LH, Fonarow GC. Clinical effectiveness of beta-blockers in heart failure: findings from the OPTIMIZE-HF (Organized Program to Initiate Lifesaving Treatment in Hospitalized Patients with Heart Failure) Registry. J Am Coll Cardiol 2009;53:184-92. [PubMed: 19130987]

29. Felker GM, Ahmad T, Anstrom KJ, et al. Rationale and design of the GUIDE-IT study: guiding evidence based therapy using biomarker intensified treatment in heart failure. J Am Coll Cardiol HF 2014;2:457-65.

30. Dunlay SM, Roger VL, Weston SA, Jiang R, Redfield MM. Longitudinal changes in ejection fraction in heart failure patients with preserved and reduced ejection fraction. Circ Heart Fail 2012; 5:720-6. [PubMed: 22936826]

31. Ather S, Chan W, Bozkurt B, et al. Impact of noncardiac comorbidities on morbidity and mortality in a predominantly male population with heart failure and preserved versus reduced ejection fraction. J Am Coll Cardiol 2012;59: 998-1005. [PubMed: 22402071]

32. Zinman B, Wanner C, Lachin JM, et al. Empa-gliflozin, cardiovascular outcomes, and mortality in type 2 diabetes. N Engl J Med 2015;373: 2117-28. [PubMed: 26378978]

33. Martens P, Mathieu C, Verbrugge FH. Promise of SGLT2 inhibitors in heart failure: diabetes and beyond. Curr Treat Options Cardiovasc Med 2017; 19:23. [PubMed: 28299616]

34. Klem I, Weinsaft JW, Bahnson TD, et al. Assessment of myocardial scarring improves risk stratification in patients evaluated for cardiac defibrillator implantation. J Am Coll Cardiol 2012; 60:408-20. [PubMed: 22835669]

35. Halliday B, Gulati A, Ali A, et al. Association between mid-wall late gadolinium enhancement and sudden cardiac death in patients with dilated cardiomyopathy and mild and moderate left ventricular systolic dysfunction. Circulation 2017;135: 2106-15. [PubMed: 28351901] 
36. Adamson PB, Abraham WT, Bourge RC, et al. Wireless pulmonary artery pressure monitoring guides management to reduce decompensation in heart failure with preserved ejection fraction. Circ Heart Fail 2014;7:935-44. [PubMed: 25286913]

37. Chow SL, Maisel AS, Anand I, et al. Role of biomarkers for the prevention, assessment, and management of heart failure: a scientific statement from the American Heart Association.

Circulation 2017;135:e1054-91. [PubMed: 28446515] 


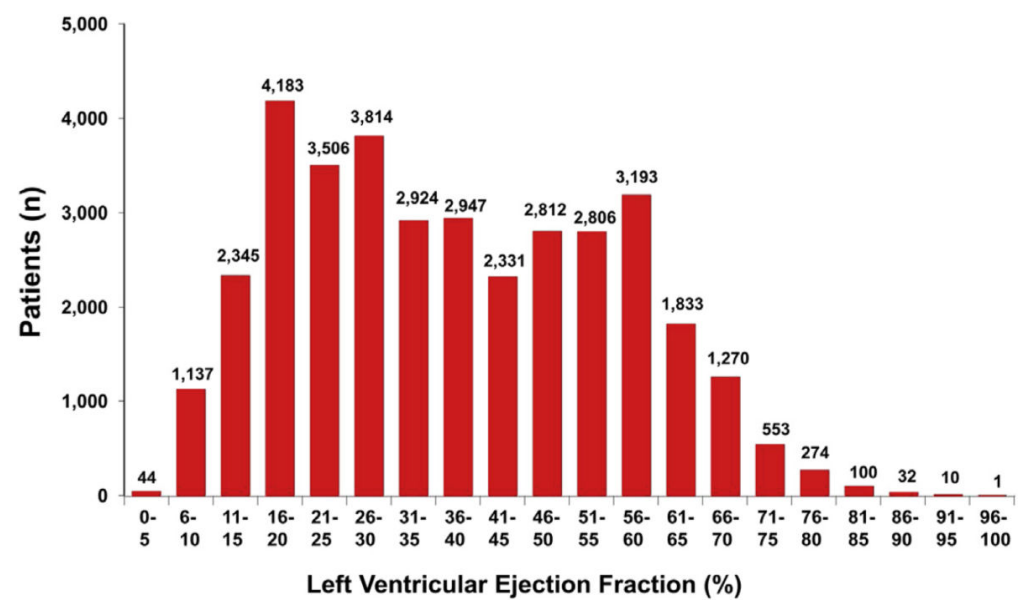

FIGURE 1. Distribution of LVEF in the OPTIMIZE-HF Study

A modest bimodal distribution of Left ventricular ejection fraction (LVEF) was observed among patients hospitalized for heart failure. OPTIMIZE-HF = Organized Program to Initiate Lifesaving Treatment in Hospitalized Patients with Heart Failure. Reprinted with permission from Fonarow et al. (2). 


\section{Outcomes in Heart Failure by LVEF Group}
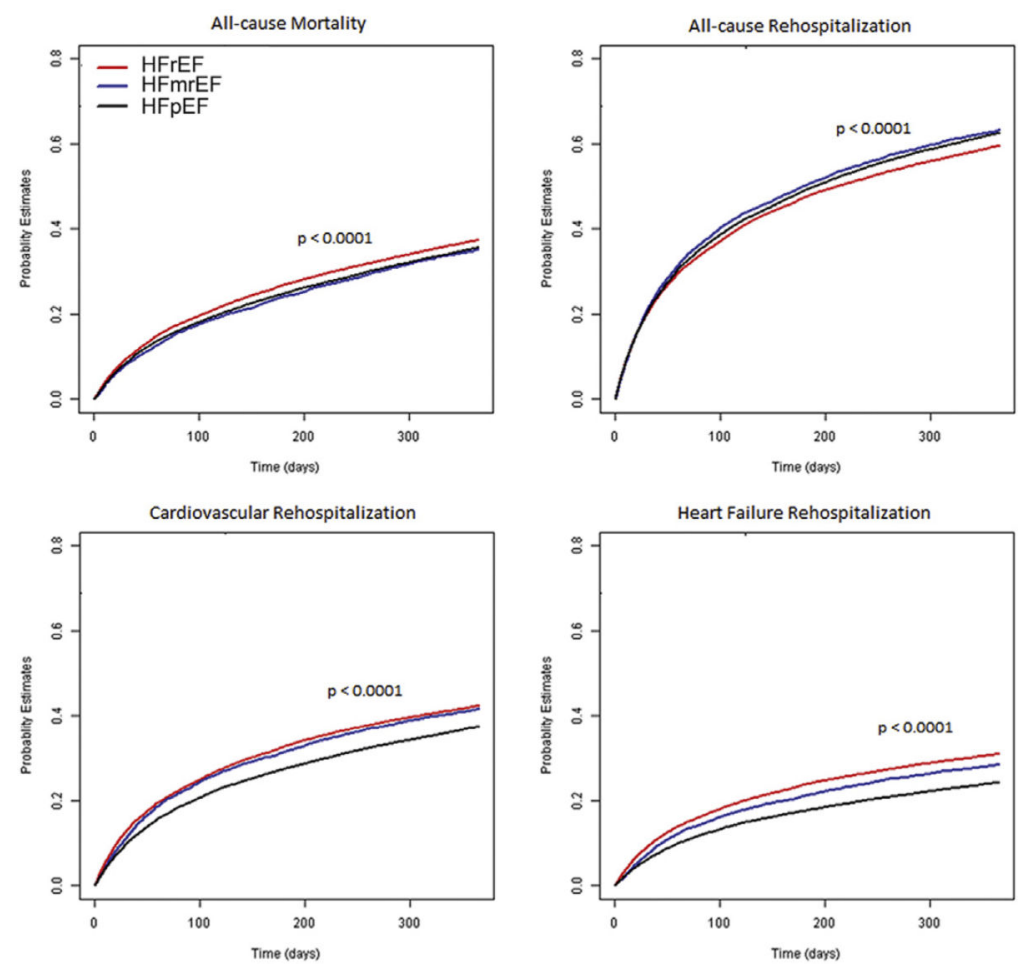

FIGURE 2. Comparisons of the Outcomes in Patients With HFpEF, HFmrEF, and HFrEF in the GWTG-HF Registry

Cumulative incidence functions for all-cause mortality, all-cause rehospitalization, cardiovascular (CV) rehospitalization, and heart failure (HF) rehospitalization in the heart failure with preserved ejection fraction (HFpEF) (black lines), heart failure with mid-range ejection fraction (HFmrEF) (blue lines), and heart failure with reduced ejection fraction $(\mathrm{HFrEF})$ (red lines) groups. GWTG-HF = Get With The Guidelines-HF Registry. Adapted with permission from Cheng et al. (8). 


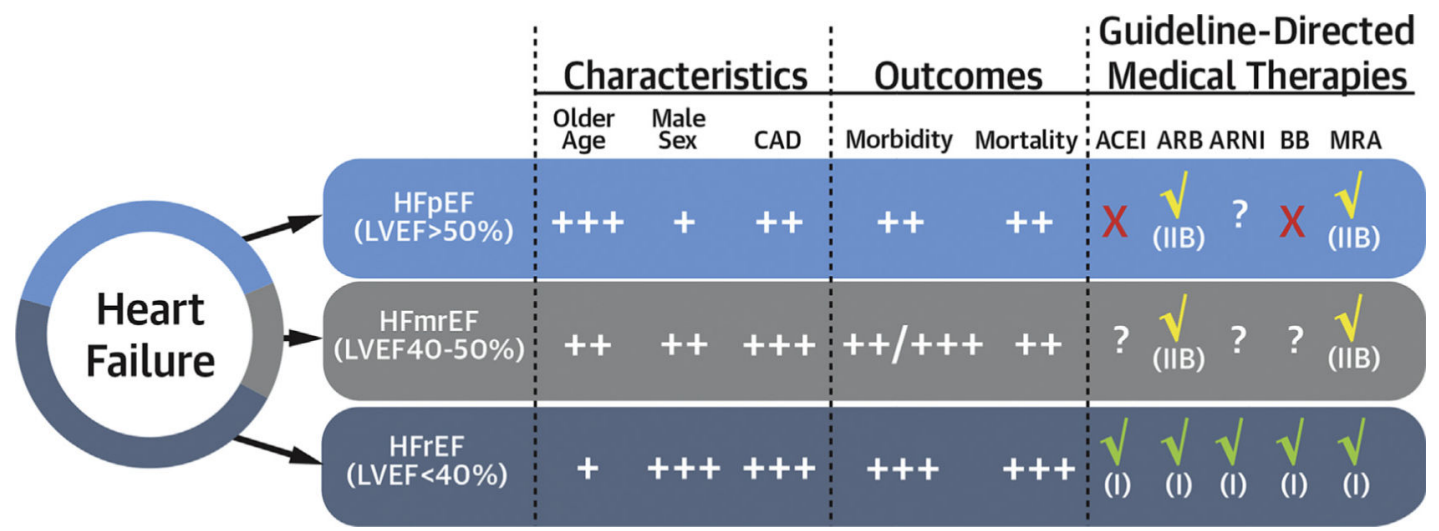

CENTRAL ILLUSTRATION. Characterization of HFpEF, HFmrEF, and HFrEF

General comparisons of the clinical characteristics, outcomes, and guideline-directed medical therapies for each heart failure group. Class of recommendation is denoted in parentheses, if applicable. ACEI $=$ angiotensin-converting enzyme inhibitors; $\mathrm{ARB}=$ angiotensin receptor blocker; $\mathrm{ARNI}=$ angiotensin receptor-neprilysin inhibitor; $\mathrm{BB}=$ betablockers; $\mathrm{CAD}=$ coronary artery disease $\mathrm{HFmrEF}=$ heart failure with mid-range ejection fraction; $\mathrm{HFpEF}=$ heart failure with preserved ejection fraction; $\mathrm{HFrEF}=$ heart failure with reduced ejection fraction; MRA $=$ mineralocorticoid receptor antagonist. 


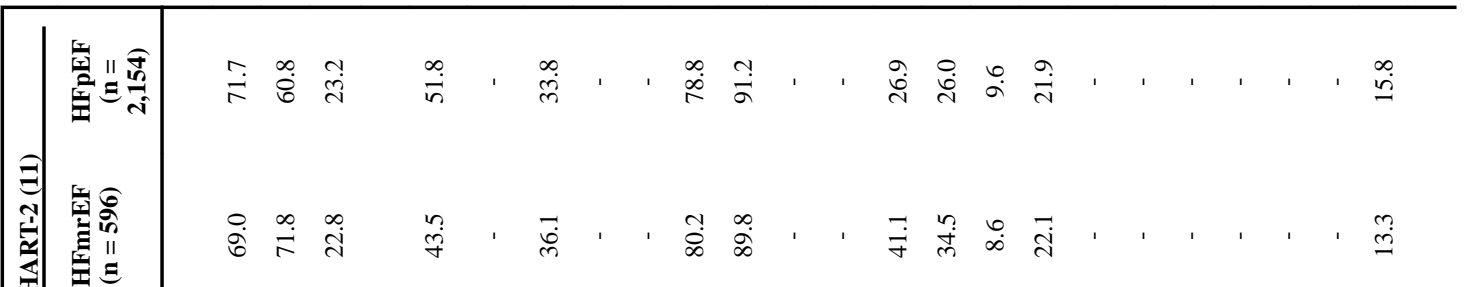

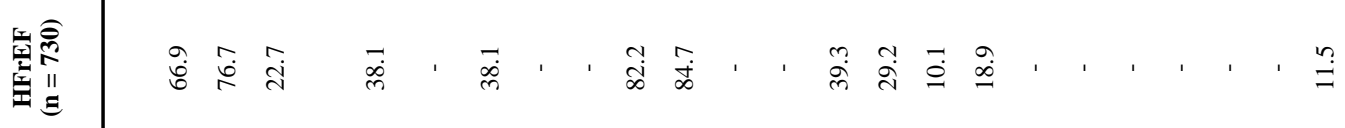

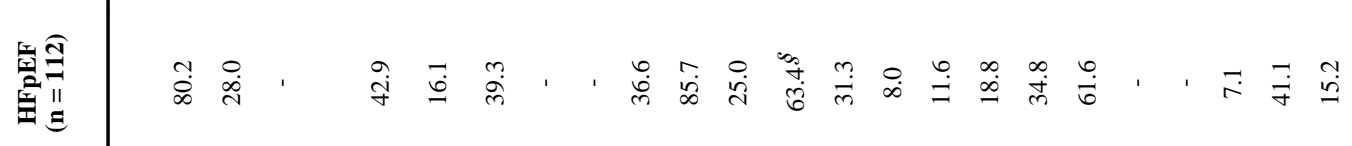




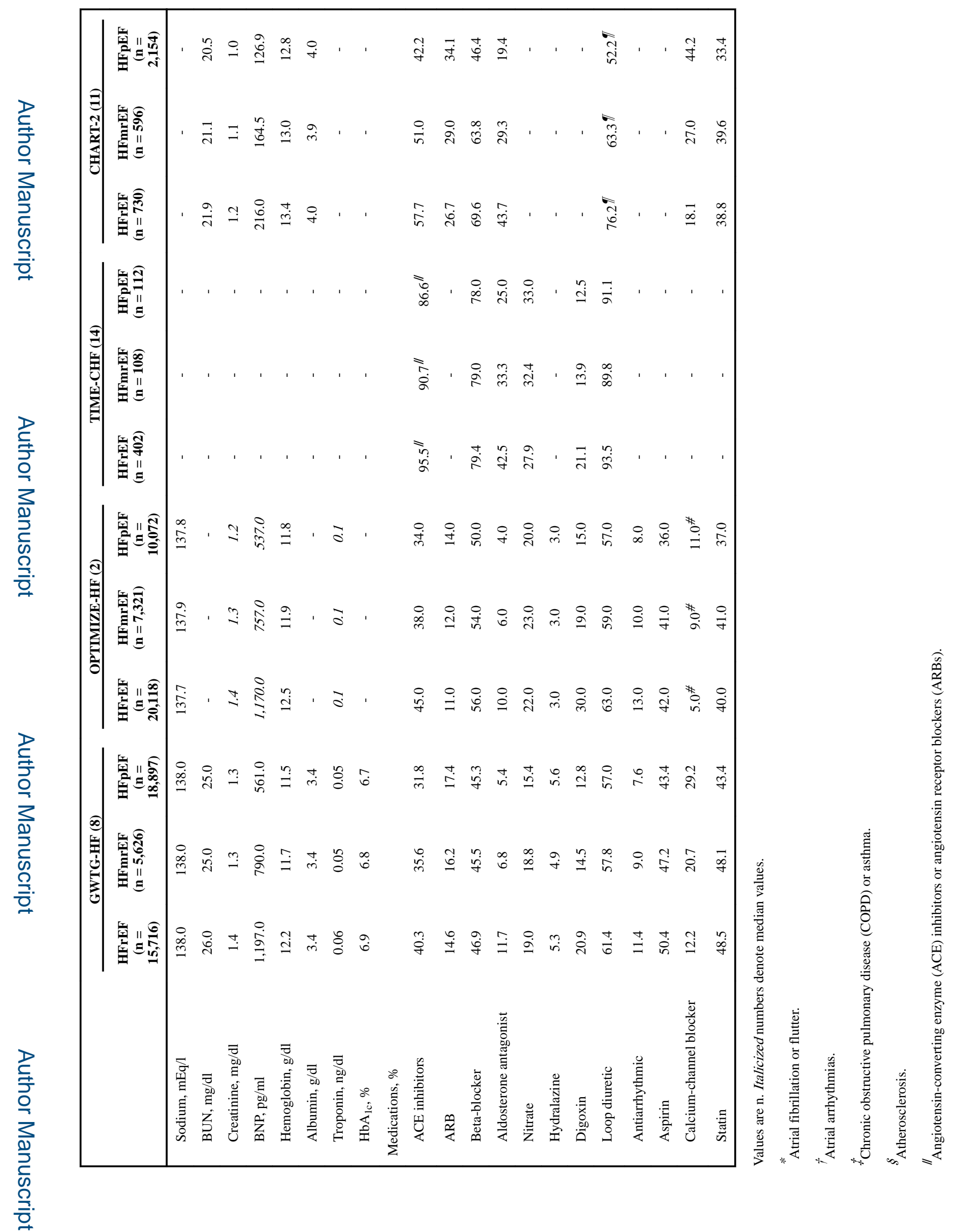

JACC Heart Fail. Author manuscript; available in PMC 2019 July 31. 


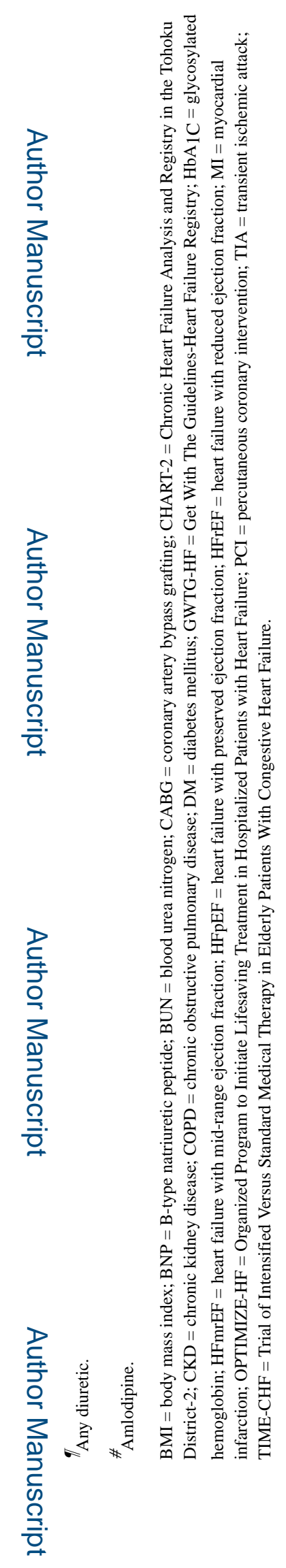

JACC Heart Fail. Author manuscript; available in PMC 2019 July 31. 